\title{
Characterization of a piezo bendable X-ray mirror
}

\section{Maurizio Vannoni, Idoia Freijo Martín, Frank Siewert, Riccardo Signorato, Fan Yang and Harald Sinn}

J. Synchrotron Rad. (2016). 23, 169-175

\section{IUCr Journals CRYSTALLOGRAPHY JOURNALS ONLINE}

Copyright (C) International Union of Crystallography

Author(s) of this paper may load this reprint on their own web site or institutional repository provided that this cover page is retained. Republication of this article or its storage in electronic databases other than as specified above is not permitted without prior permission in writing from the IUCr.

For further information see http://journals.iucr.org/services/authorrights.html 
JOURNAL OF

SYNCHROTRON

RADIATION

ISSN $1600-5775$

Received 2 July 2015

Accepted 20 October 2015

Edited by A. Momose, Tohoku University, Japan

Keywords: piezo bimorph mirror; X-ray optics; active optics; response functions; metrology.

\section{Characterization of a piezo bendable X-ray mirror}

\author{
Maurizio Vannoni, ${ }^{\mathrm{a} *}$ Idoia Freijo Martín, ${ }^{\mathrm{a}}$ Frank Siewert, ${ }^{\mathrm{b}}$ Riccardo Signorato, ${ }^{\mathrm{c}}$ \\ Fan Yang ${ }^{\mathrm{a}}$ and Harald Sinn ${ }^{\mathrm{a}}$ \\ ${ }^{\mathbf{a}}$ European XFEL, Hamburg, Germany, ${ }^{\mathbf{b}}$ Helmholtz-Zentrum Berlin-Institut für Nanometer Optic und Technologie, Berlin, \\ Germany, and ${ }^{\mathbf{C}}$ INEL Strumenti Scientifici srl, Vigonza, Italy. *Correspondence e-mail: maurizio.vannoni@xfel.eu
}

A full-scale piezo bendable mirror built as a prototype for an offset mirror at the European XFEL is characterized. The piezo ceramic elements are glued onto the mirror substrate, side-face on with respect to the reflecting surface. Using a nanometre optical component measuring machine and a large-aperture Fizeau interferometer, the mirror profile and influence functions were characterized, and further analysis was made to investigate the junction effect, hysteresis, twisting and reproducibility.

\section{Introduction}

Piezo bendable mirrors were introduced to synchrotron radiation optics in the mid-1990s as adjustable focusing or collimating elements (Signorato et al., 1998, 2001). Their ability to change focusing conditions reproducible in a short time, to enable extreme elliptical profiles and to allow to some degree wavefront corrections, made them indispensable at many synchrotron beamlines.

For X-ray free-electron lasers (XFELs), piezo bendable mirrors may become attractive for additional reasons: the coherence of the beam requires a nanometre control of the mirror shape over its entire length. Small distortions from residual mounting forces are often long range and could be neutralized by activating selected piezos. In addition, as XFEL beams are close to diffraction-limited, their footprint on the mirror is roughly proportional to the wavelength. At high power, $\mathrm{MHz}$ repetition-rate XFELs will lead at small wavelengths to a heat-bump in the centre of the mirror. Traditional mechanical benders will allow only a partial correction of this complicated and variable profile error and, therefore, a multipoint correction scheme such as piezo bendable mirrors may be advantageous.

To tune a bendable mirror effectively, it is generally required to have a full characterization in a metrology laboratory (ex situ) or directly at the X-ray beamline (in situ). Specific instruments are implemented in the first case, with particular high accuracy and a metrological calibration. The most effective instruments are the various versions of the slope-measuring profiler such as the long-trace profiler (Takacs et al., 1987), with high-accuracy modern implementations such as the nano-optic measuring machine (NOM) (Siewert et al., 2004), delivering nanometre-precision measurements with also the possibility of two-dimensional map measurement (Siewert et al., 2014). Slope-measuring profilers also provide the option to measure curved shapes of any geometry down to local radii of a few metres. The major drawback of this approach is the time needed for a complete 
measurement, still of the order of 20-30 min in the case of a long mirror of $\sim 1 \mathrm{~m}$, with a high probability of additional errors due to mirror instabilities and mechanical stresses occurring during the measurement. A different approach, mostly used at optics manufacturing sites, is Fizeau interferometry, a fast method that can deliver a two-dimensional map of the test surface in a few seconds. For this reason, this method is more effective for investigating short-term stability issues or for a slightly less precise but faster characterization of the system. One of the main limitations of this method is the final accuracy obtainable, considering that interferometric measurements are always relative to the reference flats used in the setup. This type of systematic contribution is wiped out when the difference of two measurements is performed, such as before and after the activation of a piezo. Another limitation is the minimum radius of curvature that can be inspected: in the classical 'skip-flat test' setup, or grazing-incidence setup, Fizeau interferometry is mostly suited to inspecting nominally flat mirrors rather than highly curved focusing optics (Zhang et al., 2010; Hariharan, 1997).

In this article we present a characterization of a $950 \mathrm{~mm}$ long piezo bender mirror, a prototype for a second offset mirror at the European XFEL (Sinn et al., 2011, 2012). In contrast to previous designs, where the piezo elements were buried in the mirror substrate, the piezos here are glued in groups of four to the side faces of the mirror: two on the front, close to the optical surface, and two on the back, which are polarized in the opposite direction. Each group of four piezos is driven by one channel of a high-voltage power supply (ADAPTOS high-voltage bipolar power supply from CAENels), acting as one bending element. Owing to the length of the mirror it was not possible to glue a long bar of piezo ceramic with several connectors, but instead several single elements were required. The final length was decided using a finite-element analysis (FEA) model (Yang et al., 2014). Altogether, 18 such groups of piezos were glued onto the mirror, but the first and the last two groups were connected together, so we had 16 separate channels for a fairly flexible shape correction. The piezo bender mirror is similar to that described previously (Alcock et al., 2015), but longer, i.e. $950 \mathrm{~mm}$ instead of $640 \mathrm{~mm}$ (Table 1). For a general overview of SESO technology bimorph mirrors, see also Alcock et al. (2013).

The metrology studies were carried out using two approaches: the BESSY-NOM at the Helmholtz Zentrum Berling (HZB), and a $30 \mathrm{~cm}$-diameter Fizeau interferometer at the European XFEL (XFEL-Fizeau). Both methods give an account of the overall behaviour of the system. The mirror is installed in its mechanical mount with the reflecting surface facing side-on (Siewert et al., 2012a), in the same arrangement as is supposed to be implemented in the XFEL offset mirrors system. It is polished nominally flat, and is supposed to bend in a controlled way to correct very small modifications of the surface curvature, occurring because of the heat load, or to correct the overall focal length of the beam transport system. We are considering a very long radius of curvature, so the amount of correction is extremely small, on the tens of
Table 1

Piezo bendable mirror specifications.

\begin{tabular}{ll}
\hline Dimensions $(\mathrm{L} \times \mathrm{W} \times \mathrm{H})(\mathrm{mm})$ & $950 \times 52 \times 52$ \\
Ion-beam polished area $(\mathrm{measured})(\mathrm{mm})$ & $860 \times 34$ \\
Clear aperture $(\mathrm{L} \times \mathrm{W})(\mathrm{mm})$ & $750 \times 20$ \\
Material & Silicon mono-crystal \\
Number of piezo channels & 16 \\
Quality of polishing $(\mu \mathrm{rad}$ r.m.s. $)$ & 0.3 \\
\hline
\end{tabular}

nanometres scale. For this reason we need particularly precise and sensitive measuring instruments.

We studied the piezo response functions and characterized some particular behaviours of the system including the junction effect, twisting, hysteresis and reproducibility. The general performance of this bender is evaluated and the peculiarities of an ex situ metrological characterization of very long mirrors are outlined.

\section{Measurement results with BESSY-NOM}

The BESSY-NOM instrument is a well characterized slopemeasuring instrument, delivering nanometre precise height profiles through integration of the measured slope, and it has already been used in the past to characterize bimorph mirrors (Siewert et al., 2012b). The piezo-bendable mirror is mounted in its mechanical mount, with the reflecting surface facing sideon and with the piezos connected to the power supply (Fig. 1). To avoid interference between the mechanical mount and the piezo elements attached below the mirror, the supporting points are on the end edges of the mirror. We have three supporting tips in total, two on one edge and the third one on the opposite edge. There are some lateral pushers, with

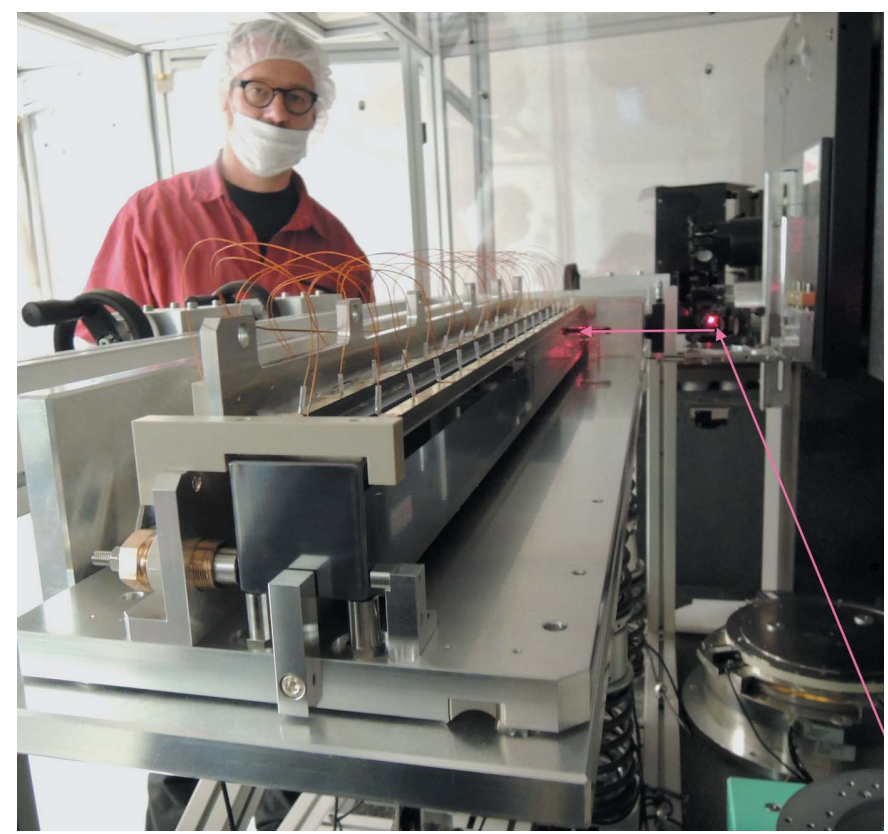

Figure 1

Photograph of the piezo bendable mirror placed in front of the BESSYNOM. The measuring beam path is sketched in red. The measurement beam is guided in the face side direction by a $45^{\circ}$ double-mirror setup, reflected with a normal angle from the mirror surface. 


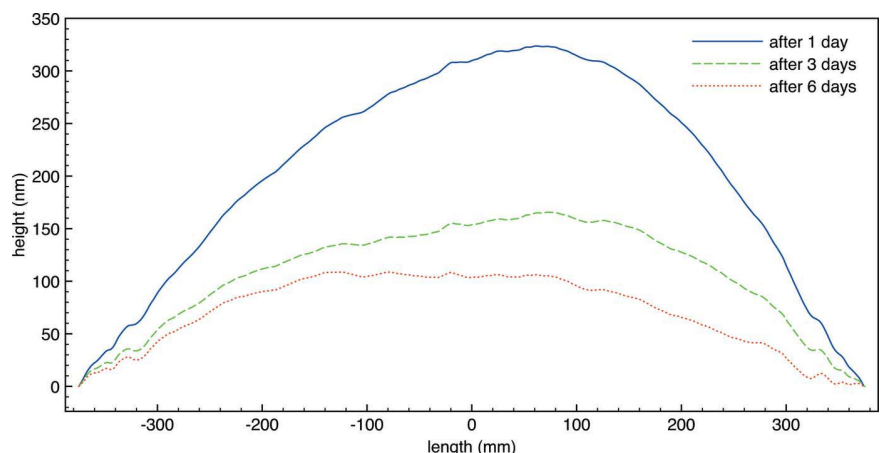

Figure 2

Profile measurements in the first week after the installation of the piezo bendable mirror in front of the BESSY-NOM.

springs, to keep the mirror in position. In principle, the mirror should not have any initial twist, because it is sitting on three points, but the situation can change during the bending because of the stress forces introduced in the mirror. The mounting situation is similar to the final installation of the mirror, only the mirror cooling part is missing: this is supposed to be implemented through a copper blade placed in contact with the mirror with indium-gallium alloy in between, so in principle it should not influence the mirror surface.

The piezo bendable mirror has been installed in its mechanical mount and then placed in front of the BESSYNOM using a kinematic table. The instrument is inside a thermally shielded tent, with temperature stability of $20 \mathrm{mK}$ over $24 \mathrm{~h}$. Despite this, we found that it took almost a week for the mirror to settle down and to become stable. In Fig. 2, we see the profile of the mirror during the first week, with the piezo still not activated. The radius of curvature changed from $223 \mathrm{~km}$ to $652 \mathrm{~km}$, convex shape. The result indicates that the mirror needs a long time to stabilize, probably because of its size and its interaction with the mechanical mount.

The shape of the mirror, mounted on the holder, was characterized with high accuracy along the central profile in face-side configuration. The mirror was also mounted on a face-up configuration, supported on a cloth, to work out a twodimensional map of the longitudinal slope (Fig. 3).

We also carried out a slope profile measurement close to the edge of the mirror surface (Fig. 4) in order to examine the junction effect.

\section{Measurement results with the XFEL-Fizeau}

The BESSY-NOM instrument can deliver line-scan and 2Dslope-map measurements in the face-up state whereas for the face-side case only line-scans can be measured at the moment. To overcome this limitation, we used a different setup at the XFEL site. The measurement is performed through a grazingincidence setup, using the XFEL large aperture Fizeau interferometer with a collimated beam of 12 inch diameter and two auxiliary reference flats (Fig. 5).

The grazing-incidence angle with respect to the surface of the mirror is about $18^{\circ}$, to minimize the desensitization due to the grazing-incidence scheme but still allowing the whole

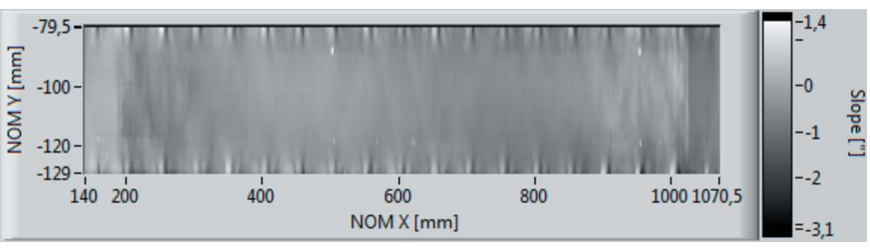

Figure 3

Longitudinal slope map of the mirror surface, placed face-up and supported on a cloth, in zero voltage state. The junction effect is present but it is limited to the outside of the clear aperture of the mirror.

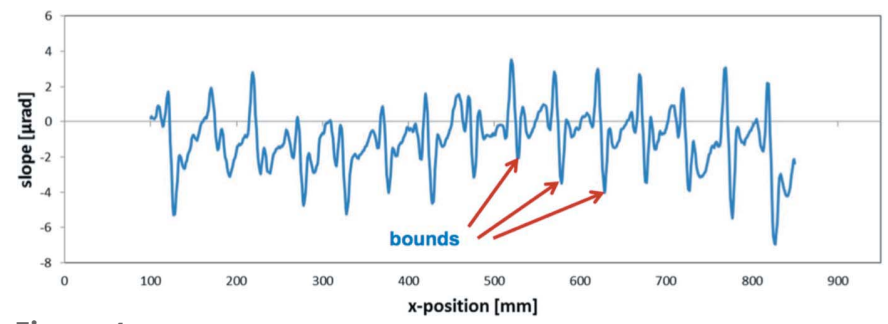

Figure 4

Slope profile scan, a few millimetres from the upper mirror edge, with evidence of the piezo bounds.

mirror to be imaged over the Fizeau clear aperture (Vannoni, 2014). The specifications of the instrument and the flats are reported in Table 2 .

Because of the grazing-incidence setup, height maps measurements have to be multiplied by the correction factor $1 /(2 \cos \alpha)$, to account for the desensitization. Then, there is a systematic error because of the non-perfect flatness of the auxiliary flats used to create the optical cavity and because of the collimation of the beam. However, in the typical piezo response function characterization, we have always to consider the subtraction between two measurements, before and after the piezo is activated, so every systematic error is removed, except air turbulence and drifting. For single measurements of the surface, the spherical contribution of the cavity can be corrected through previous calibration of the cavity without the mirror in between. With this correction, we were able to reach an agreement of better than $10 \mathrm{~nm}$ peak-tovalley (Fig. 6).

The Fizeau interferometer is using piezo-mechanical phaseshift technology, a quick method that is able to make an

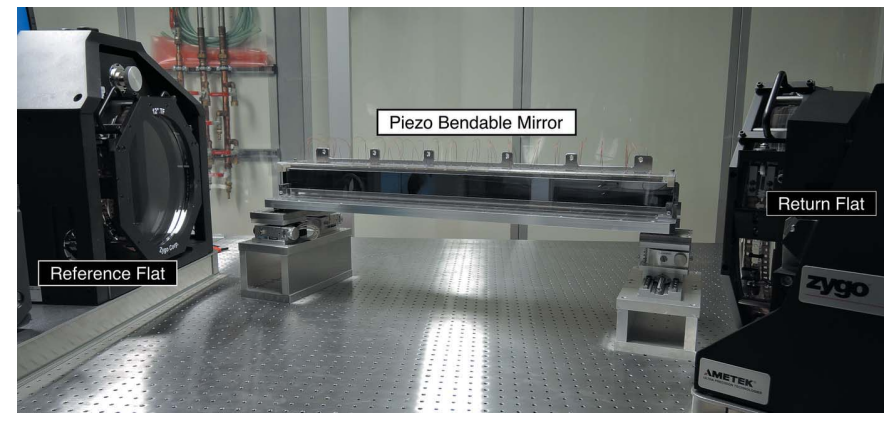

Figure 5

Photograph of the piezo bendable mirror placed in between two auxiliary optical flats, making a cavity in a grazing-incidence setup. 
Table 2

Interferometric Fizeau system and flats specifications.

\begin{tabular}{ll}
\hline Measuring principle & Phase-shift interferometry \\
Maximum aperture & $300 \mathrm{~mm}$ \\
Laser source & He-Ne laser, wavelength $\lambda=632.8 \mathrm{~nm}$ \\
Repeatability & $<0.25 \mathrm{~nm}(2 \sigma)$ \\
Resolution & $\lambda / 12000$ (high-resolution mode, double pass) \\
Camera size & $1200 \times 1200$ pixels \\
Digitization & $10 \mathrm{bits}$ \\
Reference flats clear aperture & $304.8 \mathrm{~mm}$ diameter \\
Reference flats material & Fused silica \\
Reference flats quality & $\lambda / 20$
\end{tabular}

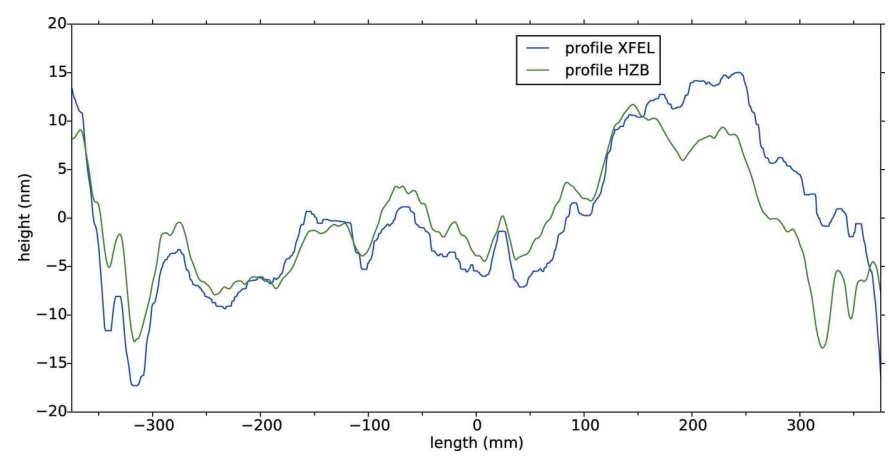

Figure 6

Central height profile of the mirror, measured by XFEL and BESSY. We removed the power contribution to account for the hysteresis effect of the piezos.

average over 200 measurements in approximately $10 \mathrm{~min}$. We need to perform a relatively high number of averages to compensate for air turbulence inside the long cavity required by the grazing-incidence setup. The instrument and the measurement optical setup are placed on an air-damped optical table to reduce environment vibrations as much as possible. The table is covered by a large clean-tent, and the measurements are performed only when the temperature has been stable within $\pm 0.1^{\circ}$ for several hours. Similarly to the BESSY-NOM in Berlin, we also found at the European XFEL that the mirror needed several days to stabilize once it had been installed in its mechanical mount in front of the interferometer (Fig. 7). In this case, the radius changed from $301 \mathrm{~km}$ to $662 \mathrm{~km}$.

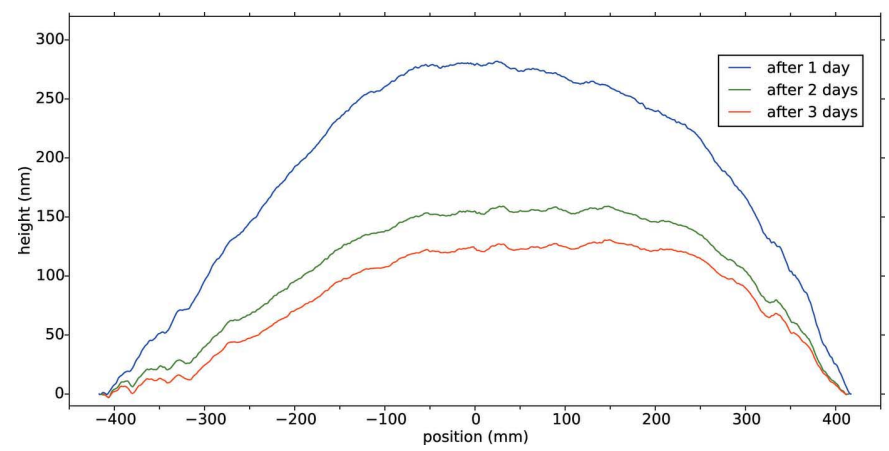

Figure 7

Profile measurements in the first days after the installation of the piezo bendable mirror in front of the XFEL-Fizeau.
The mechanical holder is supported with a stable kinematic mount with the possibility to be aligned in front of the interferometer using four translation stages to control position and orientation. The measurements are performed remotely or in an automatic way, mainly during the night or at the weekends to assure the best temperature stability (of approximately $\left.0.1^{\circ}\right)$ and fewer environmental vibrations.

\subsection{Map measurements}

Using a previous calibration of the Fizeau, we can partially compensate for the systematic errors caused by the flats and we can measure directly the two-dimensional map of the mirror surface when the piezos are activated. A typical measurement is shown in Fig. 8. We can easily see the effects of the piezos on the border, and the residual surface errors left by the ion beam polishing. The surface is imaged entirely, with small obstructions on both ends of the mirror caused by the mechanical mount, but outside the clear aperture.

An estimation of the remaining part of the unknown systematic error is about $10 \mathrm{~nm}$ peak-to-valley, as shown in Fig. 6. At this level of accuracy, we can carry out a further investigation on the junction effect that is induced by glueing the piezo elements onto the mirror. This effect was already quantified with the slope two-dimensional map at BESSY, and is also evident with the interferometric measurements (Fig. 8). If a slope map is calculated from the height map (Fig. 9), we obtain a two-dimensional map of the longitudinal map that is very similar to that shown in Fig. 3.

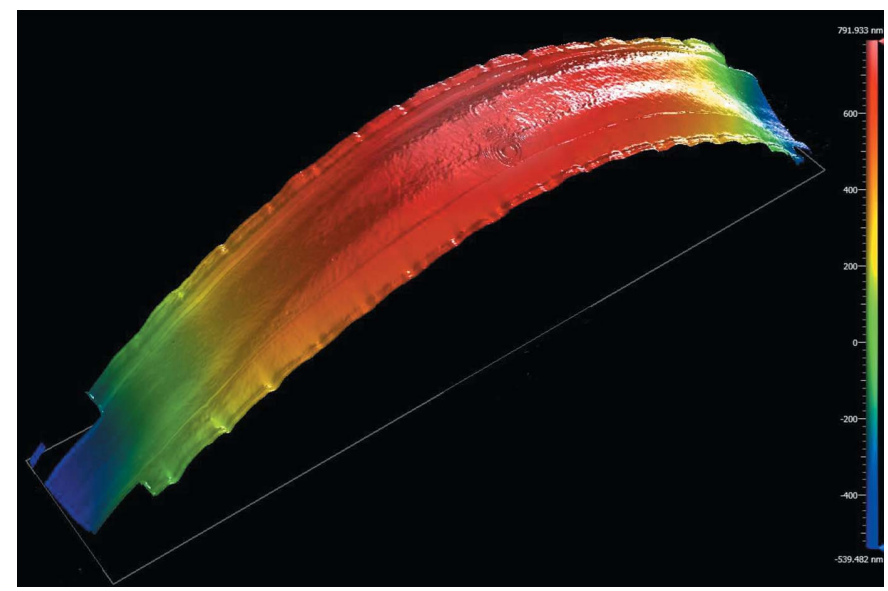

Figure 8

Full two-dimensional map of the mirror surface when it is bent by the piezos. We can see the junction effect on the mirror sides and the residual surface errors left by the ion beam polishing in the central area. These features are present even when the mirror is unbent, and do not change when bending is activated.

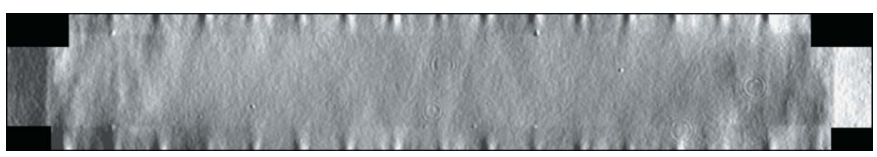

Figure 9

Longitudinal slope map of the mirror surface with all the voltages set to zero. The junction effect is evident only outside the clear aperture. 

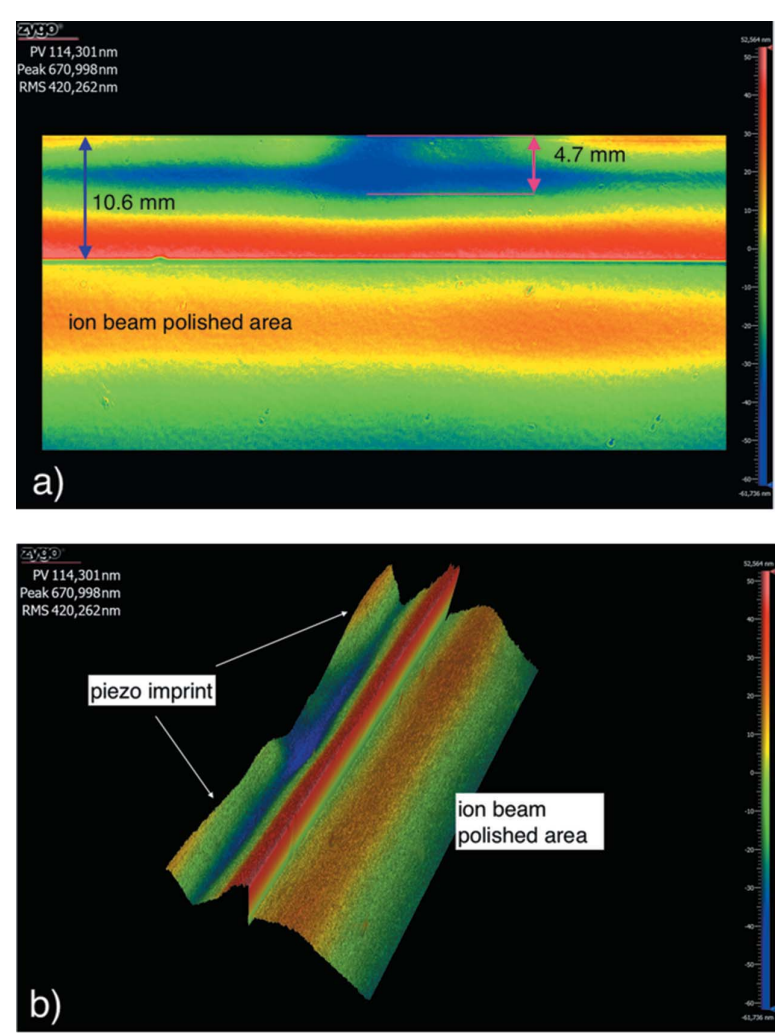

Figure 10

Height measurement of the junction area, made with a normal-incidence setup and larger zoom.

In order to investigate this effect further, we made a measurement of the mirror in the area corresponding to one junction, using the Fizeau with a smaller-diameter beam $(100 \mathrm{~mm})$ and in normal incidence. In this way we can obtain a zoomed view of the junction area with much higher resolution. The result is shown in Fig. 10. We can see that the junction effect is approximately $30 \mathrm{~nm}$ deep and appears in the surface for almost $5 \mathrm{~mm}$.

At the level of accuracy that we have, we can say that the junction effect stops before the ion-beam-polished area. The effect is caused by the particular glueing procedure and for this reason it is slightly different from junction to junction, but there are clear indications that it is always very limited. The experimental results agree with previous evaluations made using a FEA model (Yang et al., 2014).

A typical measurement of the mirror in the unbent state, with previous calibration of the cavity, shows a residual slope error of $0.36 \mu \mathrm{rad}$ r.m.s. on the central profile. This value is compatible with previous measurements performed by the BESSY-NOM and by the vendor (about $0.3 \mu \mathrm{rad}$ r.m.s.). As this is a demonstration prototype and not the final mirror, the ion beam figuring was not pushed to the limit. State-of-the-art deterministic polishing will be carried out for the final mirrors, aiming to achieve a sub-50 nrad r.m.s. slope error.

\subsection{Piezo response functions (PRFs)}

In the task of characterizing an addressable piezo bendable mirror, the most characteristic information is given by the

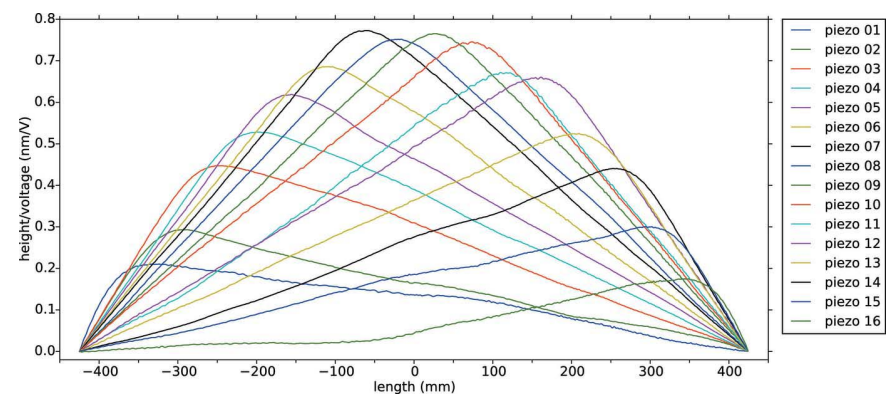

Figure 11

Piezo response functions measured activating each piezo separately.

corresponding PRFs. Assuming a fully linear regime, any induced deformation can be calculated as a linear combination of the PRFs multiplied by the particular set of piezo voltages. For this reason, having a reliable and a high-precision measurement of the PRFs is critical.

Different approaches are typically used to measure the PRFs in the case of an addressable bendable mirror. One approach is to turn on and off every piezo, in sequence, measuring the mirror before and after every single piezo is activated, and taking the difference scaled by the voltage. The PRF profile can be extracted as a one-dimensional cross section of the measured two-dimensional map. In Fig. 11 we show the result, taking the central profile over the ion-beam polished area of length $850 \mathrm{~mm}$, averaged over 200 measurements and rotated so as to have the beginning and ending points at zero for display purposes.

It is necessary to wait for a sufficient time $(30 \mathrm{~min})$ after the voltages have been applied to allow the surface to stabilize at the nanometre-level. For this reason the procedure is quite long because we need 32 measurements for 16 piezos (about $22 \mathrm{~h}$ of time in our case) and it is more sensitive to any environment variation. On the other hand, one can always monitor the zero state of the mirror after every piezo is turned off, and therefore being less sensitive to non-linearities because every piezo is activated singularly.

Another approach is to activate every piezo sequentially, without switching them off, until every piezo is charged with the same voltage. The measurements are taken every time a new piezo is activated, and the PRF corresponding to that piezo is derived by subtracting the measurements taken before and after the activation. This procedure is shorter than the previous one, because we need only 17 measurements, one at the zero state and one measurement for every piezo being activated (about $11 \mathrm{~h}$ ). In this procedure the piezos are activated together, with possible errors due to non-linearities. Despite this, the typical result reported in Fig. 12 is comparable with that obtained using the previous method. In both examples we used a voltage of $250 \mathrm{~V}$.

The PRFs measurements can be compared with FEA output using a model that has been created at XFEL (Yang et al., 2012). The simulations are reported in Fig. 13: they fit the measurements, but with some particular differences. The most evident difference is that the simulated profiles are slightly lower, meaning that the piezos are bending the mirror stronger than expected. 


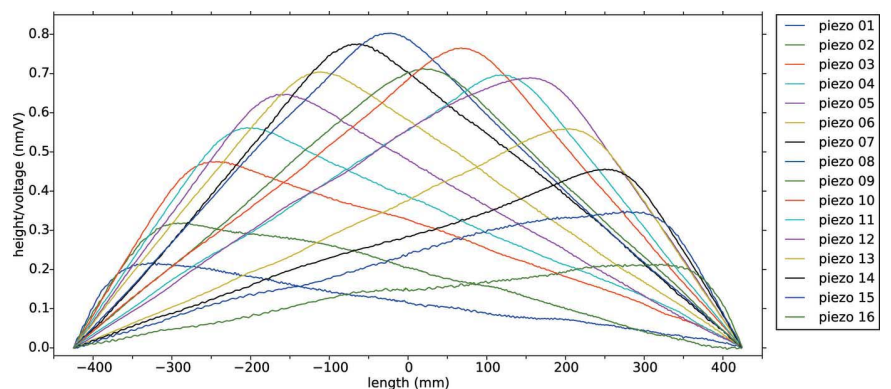

Figure 12

Piezo response functions measured activating one piezo after the other one.

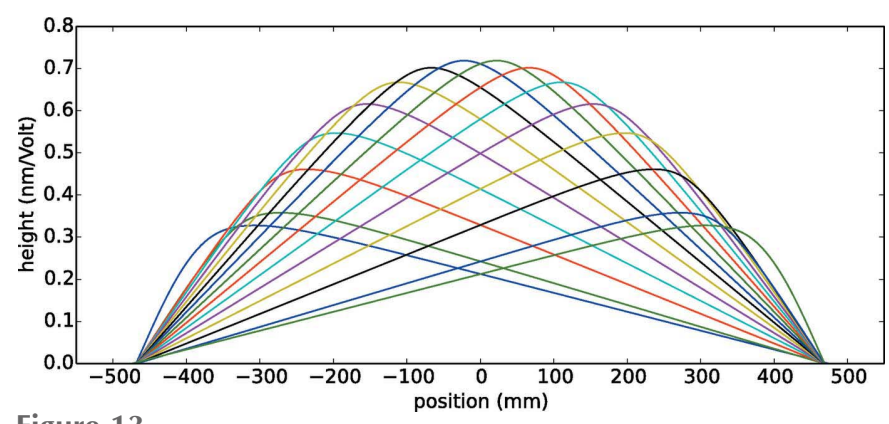

Figure 13

Piezo response functions simulated with finite-element analysis.

\subsection{Twisting}

Interferometric measurements have the great advantage of delivering a two-dimensional map of the deformation occurring in any single measurement. By studying the map, it is easy to check the details of the applied deformation, such as twisting of the induced bending. To quantify this effect, we took a linear fitting of the profiles in different positions of the clear aperture, and compared them to find the maximum peakto-valley difference. The result was always in the range of a few nanometres, so we conclude that there is no appreciable twisting introduced by the piezo actuators.

\subsection{Control of the radius of curvature}

One simple way to operate the bender is to put all the piezos at the same voltage to induce a spherical bending. In this way, small differences between the piezos are averaged out and a deformation close to a perfect sphere is expected. We tried this kind of configuration, finding very spherical profiles and an almost perfectly linear behaviour, on top of hysteresis effects. The result is shown in Fig. 14, where we measured the profile corresponding to a particular voltage and we subtracted the zero voltage state of the mirror.

\subsection{Hysteresis}

All piezoelectric materials exhibit a mechanical hysteresis as the strain does not return in the same state after charging and discharging. On top of that, there are also hysteresis effects from the mechanical mount and the glue. To quantify the overall effect on the present mirror, we made a particular sequence of measurements. We measured the mirror in the rest state, with all the piezos set to $0 \mathrm{~V}$, then we powered a

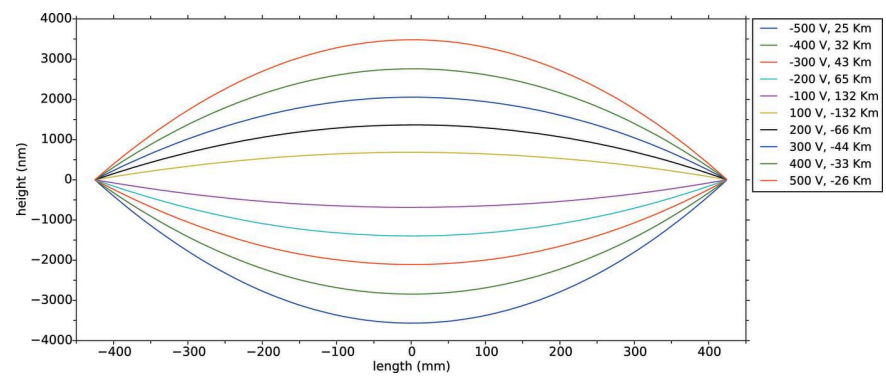

Figure 14

Change of radius of curvature activating all the piezos with the same voltage. Positive radius is for a concave surface, negative for convex. The zero voltage profile of the mirror has been subtracted.

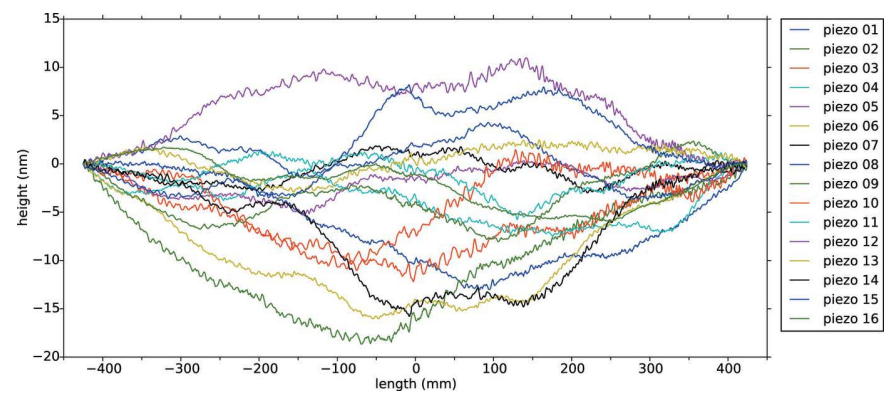

Figure 15

Hysteresis measurements obtained by measuring the difference of the measurements taken before and after a change of voltage of $250 \mathrm{~V}$.

selected piezo at $250 \mathrm{~V}$, waited half an hour and powered it again. Then, after another half an hour, we measured the mirror and subtracted the measurement from the previous one. We repeated the process for each piezo. The results are reported in Fig. 15.

These measurements were made under very stable conditions of temperature (better than $0.1^{\circ}$ of variation) and also needed a long time to be concluded.

\subsection{Stick-slip effects}

The stability of the mirror over several hours was also investigated. One piezo was activated, bending the mirror, and several measurements were recorded over time. In some cases we saw a change of curvature, randomly appearing, and we explained this effect as a friction effect of the mirror on the mechanical holder during a relaxation process after the bending. We report in Fig. 16 an example, but this phenom-

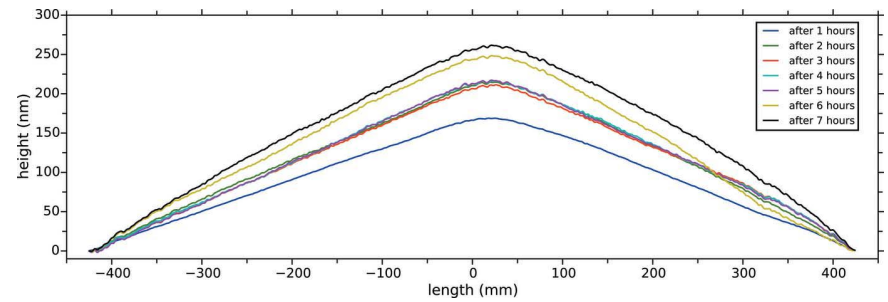

Figure 16

Measurements over a several hours time period, after a piezo was activated (No. 9). We see a change of curvature, of the order of magnitude of $1000 \mathrm{~km}$, most probably driven by a non reproducible stick-slip phenomenon. 
enon was occurring very often. More investigations are needed to understand completely the mechanism producing this change of curvature, supporting the mirror in a different way to decouple different effects. To understand the order of magnitude of the variation, on this scale $100 \mathrm{~nm}$ PV of sag corresponds to a variation in the best-fit spherical radius of $900 \mathrm{~km}$.

\section{Results and conclusions}

Despite the good results obtained in most of the measurements shown, we observed some instabilities of the measurements, even when the voltages on the piezos were kept fixed. In particular, we saw changes of curvature over a timescale of several hours, not fully correlated with the particular set of voltages that we were testing. The order of magnitude was around $50-100 \mathrm{~nm}$ of sag, that for a $1 \mathrm{~m}$-long mirror corresponds to $2500-1500 \mathrm{~km}$ radius of curvature instead of flat. We believe that this change of curvature is caused by the interaction of the mirror with the mechanical holder through the supporting points. Most probably, a stick-slip phenomenon against 'stiction' is occurring, causing random change of the radius of curvature. Because we cannot, at present, fully uncouple these effects with small modifications of the interferometer cavity, we will need to investigate this problem further. If the presence of these effects is confirmed, an improved mechanical system should be designed and tested.

The measurements show the typical behaviour of the bender, with the absence of junction effects in the central profile area and an almost perfectly spherical shape when all the piezos are activated at the same voltage. The characterization using two complete measuring systems, the BESSYNOM and the XFEL-Fizeau, agrees very well in general.

\section{Acknowledgements}

We would like to thank Luca Peverini (Thales-SESO) for fruitful technical discussions about the mirror and for performing the ion beam figuring of the surface.

\section{References}

Alcock, S. G., Nistea, I., Sutter, J. P., Sawhney, K., Fermé, J.-J., Thellièr, C. \& Peverini, L. (2015). J. Synchrotron Rad. 22, 10-15.

Alcock, S. G., Sutter, J. P., Sawhney, K. J. S., Hall, D. R., McAuley, K. \& Sorensen, T. G. (2013). Nucl. Instrum. Methods Phys. Res. A, 710, 87-92.

Hariharan, P. (1997). Opt. Eng. 36, 2478-2481.

Siewert, F., Noll, T., Schlegel, T., Zeschke, T. \& Lammert, H. (2004). AIP Conf. Proc. 705, 847-850.

Siewert, F., Buchheim, J., Boutet, S., Williams, G., Montanez, P., Krzywinski, J. \& Signorato, R. (2012a). Opt. Express, 20, 45254536.

Siewert, F., Buchheim, J., Höft, T., Fiedler, S., Bourenkov, G., Cianci, M. \& Signorato, R. (2012b). Meas. Sci. Technol. 23, 074015.

Siewert, F., Buchheim, J., Zeschke, T., Störmer, M., Falkenberg, G. \& Sankari, R. (2014). J. Synchrotron Rad. 21, 968-975.

Signorato, R., Carre, J.-F. \& Ishikawa, T. (2001). Proc. SPIE, 4501, 76 87.

Signorato, R., Hignette, O. \& Goulon, J. (1998). J. Synchrotron Rad. 5, 797-800.

Sinn, H., Dommach, M., Dong, X., La Civita, D., Samoylova, L., Villanueva, R. \& Yang, F. (2012). Technical Report XFEL EU TR2012-006. The European XFEL, Hamburg, Germany.

Sinn, H., Gaudin, J., Samoylova, L., Trapp, A. \& Galasso, G. (2011). Technical Report XFEL EU TR-2011-002. The European XFEL, Hamburg, Germany.

Takacs, S., Qian, N. \& Colbert, J. (1987). Proc. SPIE, 0749, 59-64.

Vannoni, M. (2014). Opt. Express, 22, 3538.

Yang, F., Sinn, H., Trapp, A., Signorato, R. \& Noll, T. (2012). Proceedings of MEDSI 2012, October 2012, Shanghai, China.

Yang, F., Vannoni, M., Sinn, H., Signorato, R. \& Siewert, F. (2014). Proceedings of MEDSI 2014, October 2014, Melbourne, Australia. Zhang, L., Xuan, B. \& Xie, J. (2010). Proc. SPIE, 7656, 76564W. 